\title{
KIAA1199 and its biological role in human cancer and cancer cells (Review)
}

\author{
YONGSHENG ZHANG ${ }^{1}$, SHUQIN JIA ${ }^{1,2}$ and WEN G. JIANG ${ }^{2}$ \\ ${ }^{1}$ Laboratory of Surgery, The Affiliated Hospital, Inner Mongolia Medical University, Hohhot 010050, P.R. China; \\ ${ }^{2}$ Cardiff University-Peking University Joint Cancer Institute, Cardiff University School of Medicine, \\ Heath Park, Cardiff CF14 4XN, UK
}

Received December 5, 2013; Accepted January 24, 2014

DOI: $10.3892 /$ or.2014.3038

\begin{abstract}
KIAA1199 is a gene included in the Human Unidentified Gene-Encoded (HUGE) large protein database which contains more than 2,400 members identified in the Kazusa cDNA sequencing project. Early studies described KIAA1199 as an inner ear-specific protein in which 3 point mutations were found to be associated with non-syndromic hearing loss. Recently, KIAA1199 has been found to play a central role in hyaluronan binding and depolymerisation, and a small number of studies indicate its senescence/apoptosispromoting role. An increasing body of evidence suggests its involvement in cancer progression, metastasis and poor prognosis of patients with cancer as determined in clinical studies. It has also been shown, in vitro, that KIAA1199 influences the proliferation, adhesion, motility, invasiveness and epithelialto-mesenchymal transition of cancer cells. Pathway signalling analysis found that KIAA1199 is a likely target gene of the Wnt/ $\beta$-catenin signalling pathway. Therefore, KIAA1199 may be a new and prospective target for cancer therapy, although further in vitro and in vivo investigations are required.
\end{abstract}

\section{Contents}

1. Introduction

2. KIAA1199 regulation and signalling

3. KIAA1199 and motility, invasiveness, proliferation and adhesion

Correspondence to: Professor Shuqin Jia, Laboratory of Surgery, The Affiliated Hospital, Inner Mongolia Medical University, Hohhot 010050, P.R. China

E-mail: jiashuqin2001@gmail.com

Professor Wen G. Jiang, Cardiff University-Peking University Joint Cancer Institute, Cardiff University School of Medicine, Henry Wellcome Building, Heath Park, Cardiff CF14 4XN, UK

E-mail: jiangw@cardiff.ac.uk

Key words: KIAA1199, Wnt signalling pathway, hyaluronan, cancer, metastasis, prognosis
4. KIAA1199 and human diseases

5. Conclusion

\section{Introduction}

HUGE is a database for human unidentified gene-encoded proteins larger than $50 \mathrm{kDa}$, summarising the results from the sequence analysis of human novel large cDNAs $(>4 \mathrm{~kb})$ identified in the Kazusa cDNA sequencing project (1). All genes newly characterised are conventionally identified by KIAA plus a 4-digit number. Until 2004, there were more than 2,400 entries in HUGE starting with KIAA0001, and each entry has its own gene/protein characteristic table (2). KIAA1199 was identified in 1999 (3).

KIAA1199 (Hs.459088, TMEM2L) is located on chromosome band $15 \mathrm{q} 25.1$, where a brain tumour-suppressor gene has been mapped (4). It encodes a $150-\mathrm{kDa}$ protein and was originally described as an inner ear-specific protein (5). Structurally, the KIAA1199 protein has three domains and an N-terminal secretion signal, or signal peptide, according to recently published research. The GG domain is composed of seven $\beta$-strands and two $\alpha$-helices and is $\sim 100$ amino acid residues in size. KIAA1199 has two GG domains, and the phylogenetic tree indicates that these two GG domains originated from separate combination events, instead of intragenic duplication. In the two GG domains, the $\mathrm{N}$-terminal one is more homologous to the phage gp35 proteins and Dictyostelium proteins (6). Another domain presented in the $\mathrm{N}$-terminus of KIAA1199 is called G8, containing eight conserved glycine residues and five repeated $\beta$-strand pairs. Most G8-containing proteins are predicted to be integral membrane proteins with signal peptides and/or transmembrane segments. Based on the structural and functions of G8-associated domains and proteins, it is reasonable to predict that G8 may be involved in extracellular ligand binding and catalysis processing (7).

The gene expression of KIAA1199 is tightly controlled by both genetic and epigenetic regulatory mechanisms (8). A site-direct mutagenesis study involving a series of truncated KIAA1199 promoters combined with EMSA and ChIP demonstrated the existence of $c i s$-acting elements, specifically AP-1 and NF- $\kappa \mathrm{B}$ sites, within the KIAA1199 basic promoter. The basic promoter activity of KIAA1199, however, is dependent 
on the DNA methylation status with the effective region in the first intron area of the predicted $1.9-\mathrm{kb}$ long $\mathrm{CpG}$ island $(-444$ to +1509$)$. Further analysis showed that there are two subregions in the $\mathrm{CpG}$ island based on their relatively higher GC content with respect to the rest of the island. The first subregion was identified between -444 and +280 mainly in the proximal part, and the second one was identified between +525 and +1059 in the first intron. Furthermore, the link between hypomethylation and upregulation of KIAA1199 in human breast cancer has been established. Thus, KIAA1199 is coordinately regulated through genetic and epigenetic mechanisms to control its gene expression.

KIAA1199 is an endonuclear protein and can be secreted into the extracellular environment (9). In colon cancer, strong expression of the KIAA1199 protein is localised in the cytoplasm (10), and perinuclear space (probably the ER), and the cell membrane of adenocarcinomas (11) which is different compared with gastric cancer (12) and cochlea (13) where the protein is exclusively localised in the cytoplasm.

The first publication noting KIAA1199 was a study by Abe et al (14) showing that KIAA1199 was a preferentially expressed gene in the inner ear with a $\mathrm{Cy} 3 / \mathrm{Cy} 5$ ratio of $>10$ and it was localised at 15q24 (14). Early studies have laid the foundation for more than 20 publications on human disease and the role of KIAA1199 in cell signalling, adhesion, migration and proliferation in human types of cancer.

\section{KIAA1199 regulation and signalling}

During the 10 years following the first publication relating KIAA1199 and human disease, possible signal transduction cascades involved in KIAA1199 signalling have been reported.

Wnt/ $\beta$-catenin signalling pathway. Wnt/ $\beta$-catenin signalling is a branch of an extensive functional network that developed around a class of proteins and is involved in a broad range of biological systems, including stem cell biology, developmental biology and the adult organ system (15). Wnt proteins bind to the cell surface receptors Frizzled (Fzd) and LRP5/6, thereby inhibiting glycogen synthase kinase (GSK-3 $\beta$ ) and casein kinase 1 (CK1). Inhibition of these enzymes allows the stabilization of cytoplasmic $\beta$-catenin and its eventual translocation to the nucleus, where it interacts with TCF/LEF transcription factors and induces transcription of a select subset of proteins, including PPAR $\delta$, c-myc, c-jun, cyclin D1 and ASCL2. Most colorectal cancers are caused by aberrant Wnt signalling, in which $70-80 \%$ of cases are rooted in mutational inactivation of the tumour-suppressor gene APC. This leads to stabilisation and nuclear translocation of the proto-oncogene $\beta$-catenin. Perhaps the best studied pathways of KIAA1199 is Wnt signalling in colorectal cancer.

Induction of NH2-terminal-deleted TCF4 (dominantnegative TCF4) proteins or siRNA-mediated knockdown of $\beta$-catenin in LS174 colon cancer cells is able to decrease KIAA1199 expression. The TCF4 protein is a transcription factor involved in the $\mathrm{Wnt} / \beta$-catenin signalling pathway, and findings from the genome-wide TCF4 ChIP-on-chip analyses have indicated that the KIAA1199 locus is surrounded by four TCF4-binding regions. Furthermore, KIAA1199 mRNA and protein are both confined to the proliferative compartment of normal intestinal crypts, where Wnt signalling is normally active. KIAA1199 and Wnt are highly expressed in colorectal adenomas and carcinomas, in which this pathway is almost always aberrantly activated. These features of KIAA1199 expression are also compatible with its putative role as a Wnt target gene, suggesting that KIAA1199 is a novel positively regulated target gene of the Wnt/ $\beta$-catenin signalling pathway and a putative marker of colorectal adenomatous transformation (9). The same research group, using immunoprecipitation experiments, further confirmed the interaction of KIAA1199 with the ER receptor ITPR3, a key player in $\mathrm{Ca}^{2+}$ signalling. Intracellular $\mathrm{Ca}^{2+}$ release upon ligand binding is mediated by trimeric G-proteins, which are involved in all three components of the Wnt network, i.e., the canonical $\mathrm{Wnt} / \beta$-catenin pathway, the Wnt/calcium pathway and the Wnt/Jun N-terminal kinase pathway (16-18). By modulating $\mathrm{Ca}^{2+}$ signalling, KIAA1199 is able to regulate different aspects of the Wnt and exert a negative feedback on this signalling (11). Notably, the ingenuity pathway analysis of colorectal cell line SW480 revealed that KIAA1199 knockdown affected the expression of 67 genes involved in Wnt/ $\beta$-catenin signalling; most of the genes being downregulated upon KIAA1199 knockdown. This impact was further substantiated by immunofluorescence microscopy and western blotting, showing a decreased protein expression of $\beta$-catenin and the Wnt-related stem cell marker ASCL2 (10). Together, this suggests that there may be a dual-regulation between KIAA1199 and the Wnt/ $\beta$-catenin signalling pathway. Possible crosstalks of KIAA1199 are summarised in Fig. 1.

Interactions between large KIAA proteins. To detect the protein-protein interactions between large KIAA proteins, a study based on yeast two-hybrid screening suggested that KIAA1199 may interact with KIAA0463 (plexin 2 precursor) (19). The transmembrane plexins interact with transmembrane semaphorins on nearby cells, providing 'stop' and 'go' signals that are crucial for remodelling of the cytoskeleton, cell motility and invasive growth (20). KIAA1199/plexin 2 interaction may thus play important roles in tumourigenesis and the invasiveness of cells.

COX2 inhibitor NS398 attenuates KIAA1199 expression. Elevated COX2 levels may lead to tumour development and progression through activation of the EGFR and Tcf/Lef signal transduction pathways. N-(2-cyclohexyloxy-4-nitrophenyl)methanesulfonamide (NS398), a selective COX2 inhibitor, exerts its anticarcinogenic effect by inducing apoptosis (21), inhibiting cell cycle progression, angiogenesis (22) metastasis (23) and enhancing the effect of antitumour agents (24). Dose-dependent treatment of NS398 had a reverse effect on the expression of genes increased in the colorectal normaladenoma sequence in HT29 colon adenocarcinoma cells, including KIAA1199 (25), although the mechanism is not yet clear.

\section{KIAA1199 and cellular motility, invasiveness, proli- feration and adhesion}

KIAA1199 has been shown to be involved in several human diseases or conditions that are marked by aberrant cell migration and proliferation. Cell culture studies demonstrated a 
A

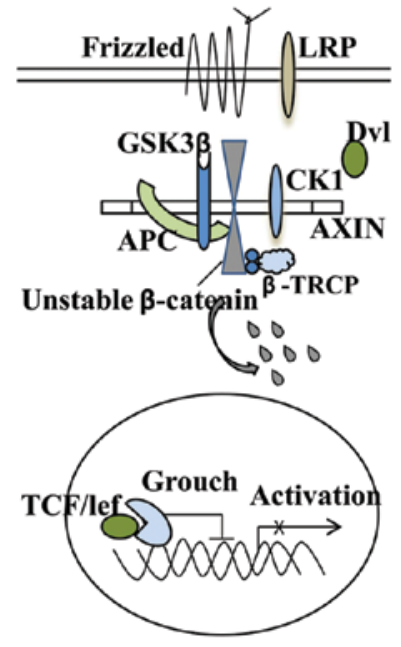

B

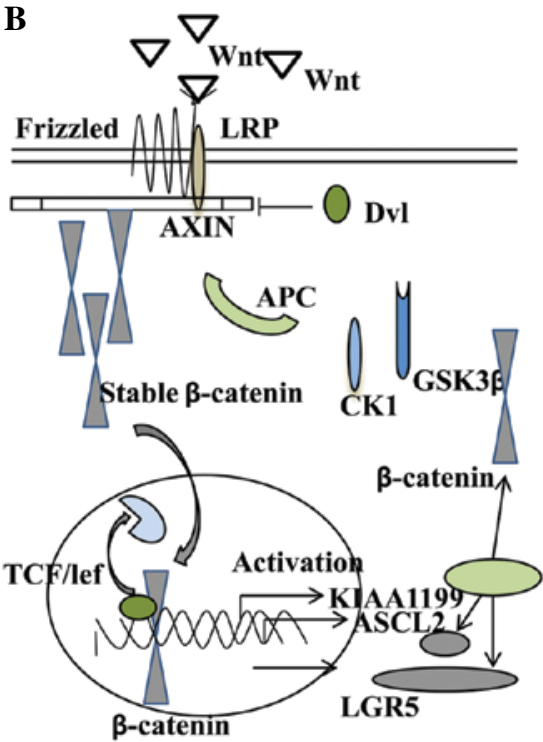

C

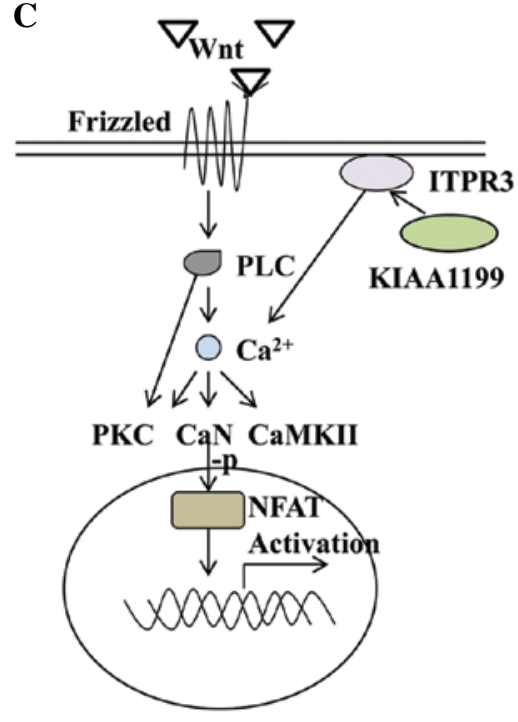

Figure 1. Representative scheme of the KIAA1199 and Wnt/ $\beta$-catenin signalling pathway network. (A) Transcription of Wnt target genes is not activated in the absence of Wnt (canonical Wnt pathway). (B) KIAA1199 is a positively regulated target gene in the presence of Wnt signalling. Inversely, KIAA1199 can feedback-control the genes of the Wnt/ $\beta$-catenin signalling pathway such as $\beta$-catenin, ASCL2 and LGR5. (C) In the presence of KIAA1199, by interacting with ITPR3 and modulating $\mathrm{Ca}^{2+}$ signalling, KIAA1199 may exert a negative feedback on $\mathrm{Wnt} / \mathrm{Ca}^{2+}$ signalling.

potential biological role for KIAA1199 in the regulation of cell motility, invasiveness, proliferation and adhesion.

Motility, invasiveness and metastasis. Cell migration is a critical determinant that precedes proliferation in the pathogenesis of a number of diseases, including cancer, atherosclerosis, asthma and many others. The role of KIAA1199 in promoting cell motility, invasiveness and metastasis has been demonstrated in a number of different cell types.

In colorectal cancer SW480 cells, KIAA1199 knockdown was found to reduce the actin filament or microtubule-based cellular movement involving genes such as MYO1E, KIF1B or TUBE1 as well as the maintenance of cytoskeleton signalling involving genes such as MTSS1, ARHGAP26, DIAPH2, ABLIM1 or DAAM1. Real-time cell analysis (RTCA)-based cellular migration analysis demonstrated decreased migration upon KIAA1199 knockdown. Moreover, ingenuity pathway analysis showed that molecules modulating cell-cell contacts were altered upon KIAA1199 knockdown (10). In breast cancer MDA-MB-435 cells, silencing of KIAA1199 resulted in mesenchymal-epithelial transition (MET) that reduced cell migratory ability in vitro and decreased metastasis in vivo. Gain-offunction assays further demonstrated the role of KIAA1199 in promoting cell migration. KIAA1199-enhanced cell migration was found to require endoplasmic reticulum (ER) localization, where it forms a stable complex with the chaperone binding immunoglobulin protein (BiP). A novel ER-retention motif within KIAA1199 was identified and is known to be required for its ER localization, interaction with ER glucose-regulated protein 78/binding immunoglobulin protein (GRP-78/BiP) and enhancement of cellular migration. The KIAA1199-mediated cellular migration was hypothesised to be due to KIAA1199mediated ER calcium leakage and the resultant increase in cytosolic calcium ultimately leading to protein kinase $\mathrm{C} \alpha$ activation and subsequent cell migration (26).
Proliferation. Proliferation analysis using RTCA and MTT demonstrated that knockdown using three different constructs reduced the proliferation rate of colorectal cancer cells by $\sim 50 \%$ as well as a decrease in the proliferation marker Ki-67 and phospho-Rb (10). Knockdown of KIAA1199 decreased the proliferation of gastric cancer cell lines AGS and HGC27 (27).

Adhesion. Using colorectal cancer SW480 cells, RTCA demonstrated that knockdown of KIAA1199 led to a 50\% reduced cellular adhesion. Decreased adhesion was confirmed by a colorimetric assay on fibronectin-coated plates (10). In colorectal cancer, mutational inactivation of the APC led to the stabilisation and nuclear translocation of the proto-oncogene $\beta$-catenin, which has a crucial role in cell-cell adhesion. KIAA1199 knockdown was found to attenuate the effects of Wnt/ $\beta$-catenin signalling, thus attenuating the adhesion of SW480 cells.

\section{KIAA1199 and human diseases}

KIAA1199 is expressed in a wide range of normal human tissues, with the highest level of expression noted in brain. Mutated KIAA1199 was shown to be linked to systematic hearing loss. Dysregulated expression of mature KIAA1199 has been frequently detected in cancers and senescencerelated diseases. In addition, immature KIAA1199 with the $\mathrm{N}$-terminal signal peptide was found to be involved in the metabolism of hyaluronan (HA), although the basic function of KIAA1199 remains unknown.

Hereditary hearing loss. Hereditary hearing loss is a highly heterogeneous sensory disorder in genetic terms. By cDNA microarray analysis, KIAA1199 was found to be highly expressed in the inner ear (14). In situ hybridisation findings found that KIAA1199 was expressed in Deiters' cells and/or 
the spiral ligament and has three possible non-syndromic hearing loss-causing point mutations of which an R187C mutation in one family (located at the $\mathrm{N}$-terminal GG domain), an R187H mutation in two unrelated families, and an $\mathrm{H} 783 \mathrm{Y}$ mutation in one sporadic case of non-syndromic hearing loss were noted. Although the R187C and R187H mutations did not appear to affect cytoplasmic localisation of the gene product in vitro, the H783Y mutation showed an irregular and worm-eaten pattern, that may underlie the molecular mechanism of hearing impairment (5). However, Usami et al found that in a Japanese population, mutations in GJB2, SLC26A4 and CDH23, and the mitochondrial $12 \mathrm{~S}$ rRNA, are the major causes of hearing loss. Mutations in KIAA1199 and several other genes have been noted in independent autosomal dominant families (28). Furthermore, Usami et al demonstrated the distribution of KIAA1199 in Deiters' cells, as well as in various supporting cells in the organ of Corti including border, inner phalangeal, and inner and outer pillar cells, although the detailed function of KIAA1199 protein in these cells remains unknown (13).

Keratoconus with cataract. To investigate the fine mapping of the keratoconus with cataract locus on chromosome $15 q$ and the mutational analysis of positional candidate genes, Dash et al carried out the genotyping of two novel microsatellite markers and a single nucleotide polymorphism (SNP) in the critical region of linkage for keratoconus with cataract on 15q (29). KIAA1199 was found to be one of the positional candidate genes for mutation (29).

Gastric cancer. KIAA1199 was found to be highly expressed in gastric cancer, and has been associated with prognosis and lymph node metastasis in multivariate analyses (12). Chivou Economescu et al found that KIAA1199 was one of the seven genes that was significantly upregulated in gastric cancer and was associated with gastric cancer progression (30). Jia et al (27) found that high levels of KIAA1199 expression in gastric cancer patients were noted in advanced stages and poorer prognosis, thus indicating a potential prognostic value.

Colorectal tumours. Expression of KIAA1199 has been extensively studied in colorectal tumours with differing expression profiles. In normal mucosa, KIAA1199 protein expression has been shown to be confined to cells in the lower portion of intestinal crypts, where Wnt signalling is physiologically active, but is markedly increased in adenomas $(9,25)$, colorectal cancer $(11,31)$ and stage I-IV adenocarcinomas (10). However, Galamb et al discovered higher expression of KIAA1199 only in adenoma and not in colorectal cancer, when compared to that in normal colorectal tissue, suggesting a possible early role of KIAA1199 in the oncogenesis of colorectal cancer. Array-based methylation analysis and comparison with transcript expression data has provided additional evidence that KIAA1199 expression may be shut down in normal colon mucosa by promotion or methylation (10). KIAA1199 mRNA was detected in the plasma of approximately $80 \%$ of individuals with colorectal adenomas or cancers. Unfortunately, it was also identified in $30 \%$ of tumour-free controls, thus the ultimate diagnostic value remains to be determined (31).
Breast cancer. Kuscu et al found that KIAA1199 was upregulated in invasive breast cancer specimens and invasive MDA-MB-231 breast cancer cells whereas minimal expression was noted in non-invasive MCF-7 breast cancer cells (8), indicating a role of KIAA1199 in the invasive potential of cancer cells. In separate studies, two breast cancer cell lines, T-47D and ZR-75-1, with low invasive potential, expressed virtually a negative amount of KIAA 1199 mRNA whereas the highly invasive cell lines (MDAMB-435 and MDA-MB-231) expressed higher levels of KIAA1199. This again suggests that the upregulation of KIAA1199 may play a role in the development of some, but not all, breast cancers. Another possibility is that MDA-MB-435 and MDA-MB-231 cells have a mutation in the KIAA1199 gene $(4,26)$.

Familial glioma. Importantly, the KIAA1199 gene is located on chromosome $15 \mathrm{q} 25$, where putative tumour-suppressor genes of various human cancers are located (e.g. brain tumours, ovarian and small cell lung carcinomas) (32-34). Of particular interest is that the KIAA1199 gene lies within the chromosomal region showing a strong linkage to familial gliomas (32). Given that the brain is the organ with the highest expression of KIAA1199, investigation of whether KIAA1199 plays a role in the development of familial and sporadic gliomas is warranted.

Oral squamous cell carcinoma (OSCC). Using two-dimensional (2D) SDS-PAGE electrophoresis accompanied by mass spectrometry, 10 pairs of OSCCs and adjacent non-tumour tissues from 5 cases of early-stage and 5 cases of late-stage OSCCs were examined to identify differentially expressed genes. KIAA1199 was found to be highly expressed in both early- and late-stage OSCCs, indicating that KIAA1199 may be a novel marker and may be involved in the oncogenesis of OSCC (35).

Uterine leiomyosarcomas (ULMS). A genome-wide arraybased comparative genomic hybridisation (array-CGH) analysis method was used to analyse 15 cases of ULMS in order to identify novel genes following genomic DNA copy number changes. KIAA1199 was one of the representative frequently gained BAC clones (36), which suggests a new regulation mode for KIAA1199 expression.

Prostate cancer. Minimal expression of KIAA1199 was found in normal prostate epithelial PrEC cells (4) and in noninvasive LNCaP prostate cancer cells. However, high levels of KIAA1199 were observed in invasive DU145 prostate cancer cells (8), again indicating that KIAA1199 may be correlated with the progression and invasiveness of prostate cancer cells.

Tumour-related senescence. In a study concerning the mutual paracrine interactions of human mesenchymal stem cells (hMSCs) and glioblastoma multiforme (GBM) cells under in vitro co-culture conditions compared with their monocultures, Motaln et al (37) showed that hMSCs were responsible for the impairment of GBM cell invasion and growth, possibly via induction of their senescence. They found CCL2/MCP-1 and other deregulated chemokines may account senescence for the altered cocultured cell phenotype by affecting genes asso- 
ciated with proliferation, invasion and senescence including KIAA1199 and SerpinB2.

To identify the genes involved in chromosome 3-induced cellular mortality, Michishita et al (4) performed a cDNA subtraction experiment using immortal renal cell carcinoma cells (RCC23) and the mortal counterpart with the transferred chromosome 3 (RCC23+3). A striking upregulation of KIAA1199 mRNA in mortal RCC23+3 was found. Taking the DNA localisation into account, where a brain tumoursuppressor gene has been mapped, the findings suggest that the KIAA1199 gene may play a role in cellular mortality, and replicative senescence of normal human cells, which counter cell immortalisation and carcinogenesis.

In addition, KIAA1199 was also highly expressed in neurally cultured human skeletal muscle cells when compared with that in tissue biopsies (38). Cultured muscle cells displayed reductive metabolic and muscle-system transcriptome adaptations as observed in muscle atrophy, and they activated tissue-remodelling and senescence/apoptosis processes. Based on the role of KIAA1199 in cellular mortality, this suggests that cultured muscle cells had activated a senescence process that was not established in the muscle tissues. Further IPA analysis revealing apoptosis as a regulated signalling pathway supported this point.

Hyaluronan metabolism. Hyaluronan (HA) has an extraordinarily high output in tissues such as skin, cartilage and other connective tissues, providing structural and functional integrity to organs. HA degradation is accelerated in inflammatory diseases and cancers. CD44 (39) and two hyaluronidases (HYAL1 and HYAL2) (40) are thought to be responsible for HA binding and degradation. In a study by Yoshida et al, using glycosaminoglycan-binding assays, KIAA1199 was found to play a central role in HA binding and depolymerisation that was independent of CD44 and HYAL enzymes (41). Knockdown of KIAA1199 attenuated HA degradation by human skin fibroblasts, and transfection of KIAA1199 cDNA into cells conferred the ability to catabolise HA in an endo- $\beta$-N-acetylglucosaminidase-dependent manner via the clathrin-coated pit pathway. Increased degradation of HA in synovial fibroblasts from patients with osteoarthritis or rheumatoid arthritis was correlated with increased levels of KIAA1199 and was abrogated by the knockdown of KIAA1199. The level of KIAA1199 in uninflamed synovium was less than that in osteoarthritic or rheumatoid synovium. These data suggest that KIAA1199 is a unique hyaladherin with an important role in HA catabolism in the dermis of the skin and arthritic synovium. Recently, Yoshida et al disclosed that a murine homologue of human KIAA1199 (mKiaa1199) selectively catabolised HA via the clathrin-coated pit pathway. A glycosaminoglycan-binding assay demonstrated the specific binding of mKiaa1199 to HA, although slight differences were found in the peak sizes of the minimum degradates of HA (42). Together, it is suggested that, similar to hKIAA1199, mKiaa1199 is also a hyaladherin, leading to HA depolymerisation. Yoshida et al carried out further research on KIAA1199 and hyaluronan metabolism. They found that cleavage of $\mathrm{N}$-terminal 30 amino acids occurs in functionally matured KIAA1199 resulting in altered intracellular trafficking of the molecule and loss of cellular HA depolymerisation. This suggests that the N-terminal portion of KIAA1199 functions as a cleavable signal sequence required for proper KIAA1199 translocation and KIAA1199-mediated HA depolymerisation. Notably, the secreted mature-form of KIAA1199 showed no HA degrading activity, together supporting the idea that KIAA1199-mediated HA depolymerisation occurred through rapid vesicle endocytosis $(41,43)$.

\section{Conclusion}

KIAA1199 is a newly found gene with significance in several research fields, including hereditary hearing loss, hyaluronan metabolism, senescence and cancer. KIAA1199 accelerates senescence/apoptosis in certain situations. KIAA1199 generally behaves as an oncogene that is highly expressed in different types of cancers and has been correlated with the progression, distant metastasis and poor prognosis of cancer patients. In vitro, KIAA1199 stimulates proliferation, adhesion, motility, invasiveness and epithelial-to-mesenchymal transition of cancer cells. Pathway signalling analysis indicates that it is a target gene of canonical Wnt/ $\beta$-catenin signalling and by regulating $\mathrm{Ca}^{2+}$, KIAA1199 can feedback-control all of the three components of $\mathrm{Wnt} / \beta$-catenin signalling, thus providing a new and promising target for broad-spectrum cancer therapy.

\section{Acknowledgements}

The authors wish to thank the Cancer Research Wales and the Albert Hung Foundation for supporting the present study. This study was also supported by the 2013 National Natural Science Foundation of China (no. 81260363).

\section{References}

1. Suyama M, Nagase T and Ohara O: HUGE: a database for human large proteins identified by Kazusa cDNA sequencing project. Nucleic Acids Res 27: 338-339, 1999.

2. Kikuno R, Nagase T, Nakayama M, et al: HUGE: a database for human KIAA proteins, a 2004 update integrating HUGEppi and ROUGE. Nucleic Acids Res 32: D502-D504, 2004.

3. Nagase T, Ishikawa K, Kikuno R, Hirosawa M, Nomura N and Ohara O: Prediction of the coding sequences of unidentified human genes. XV. The complete sequences of 100 new cDNA clones from brain which code for large proteins in vitro. DNA Res 6: 337-345, 1999.

4. Michishita E, Garcés G, Barrett JC and Horikawa I: Upregulation of the KIAA1199 gene is associated with cellular mortality. Cancer Lett 239: 71-77, 2006.

5. Abe S, Usami S and Nakamura Y: Mutations in the gene encoding KIAA1199 protein, an inner-ear protein expressed in Deiters' cells and the fibrocytes, as the cause of nonsyndromic hearing loss. J Hum Genet 48: 564-570, 2003.

6. Guo J, Cheng H, Zhao S and Yu L: GG: a domain involved in phage LTF apparatus and implicated in human MEB and nonsyndromic hearing loss diseases. FEBS Lett 580: 581-584, 2006.

7. He QY, Liu XH, Li Q, Studholme DJ, Li XW and Liang SP: G8: a novel domain associated with polycystic kidney disease and nonsyndromic hearing loss. Bioinformatics 22: 2189-2191, 2006.

8. Kuscu C, Evensen N, Kim D, Hu YJ, Zucker S and Cao J: Transcriptional and epigenetic regulation of KIAA1199 gene expression in human breast cancer. PLoS One 7: e44661, 2012.

9. Sabates-Bellver J, Van der Flier LG, de Palo M, et al: Transcriptome profile of human colorectal adenomas. Mol Cancer Res 5: 1263-1275, 2007.

10. Birkenkamp-Demtroder K, Maghnouj A, Mansilla F, et al: Repression of KIAA1199 attenuates Wnt-signalling and decreases the proliferation of colon cancer cells. Br J Cancer 105: $552-561,2011$. 
11. Tiwari A, Schneider M, Fiorino A, et al: Early insights into the function of KIAA1199, a markedly overexpressed protein in human colorectal tumors. PLoS One 8: e69473, 2013.

12. Matsuzaki S, Tanaka F, Mimori K, Tahara K, Inoue $\mathrm{H}$ and Mori M: Clinicopathologic significance of KIAA1199 overexpression in human gastric cancer. Ann Surg Oncol 16: 2042-2051, 2009.

13. Usami S, Takumi Y, Suzuki N, et al: The localization of proteins encoded by CRYM, KIAA1199, UBA52, COL9A3, and COL9A1, genes highly expressed in the cochlea. Neuroscience 154: 22-28, 2008.

14. Abe S, Katagiri T, Saito-Hisaminato A, et al: Identification of $C R Y M$ as a candidate responsible for nonsyndromic deafness, through cDNA microarray analysis of human cochlear and vestibular tissues. Am J Hum Genet 72: 73-82, 2003.

15. Voronkov A and Krauss S: Wnt/beta-catenin signaling and small molecule inhibitors. Curr Pharm Des 19: 633-664, 2013.

16. Katanaev VL, Ponzielli R, Sémériva $M$ and Tomlinson A: Trimeric $\mathrm{G}$ protein-dependent frizzled signaling in Drosophila. Cell 120: 111-122, 2005.

17. Ma L and Wang HY: Suppression of cyclic GMP-dependent protein kinase is essential to the Wnt/cGMP/Ca ${ }^{2+}$ pathway. J Biol Chem 281: 30990-31001, 2006.

18. Kestler HA and Kühl M: From individual Wnt pathways towards a Wnt signalling network. Philos Trans R Soc Lond B Bio Sci 363: 1333-1347, 2008

19. Nakayama M, Kikuno R and Ohara O: Protein-protein interactions between large proteins: two-hybrid screening using a functionally classified library composed of long cDNAs. Genome Res 12: 1773-1784, 2002.

20. Zachary IC, Frankel P, Evans IM and Pellet-Many C: The role of neuropilins in cell signalling. Biochem Soc Trans 37: 1171-1178, 2009.

21. Ramer R, Walther U, Borchert P, Laufer S, Linnebacher M and Hinz B: Induction but not inhibition of COX-2 confers human lung cancer cell apoptosis by celecoxib. J Lipid Res 54: 3116-3129, 2013.

22. Abdelrahim M and Safe S: Cyclooxygenase- 2 inhibitors decrease vascular endothelial growth factor expression in colon cancer cells by enhanced degradation of $\mathrm{Sp1}$ and $\mathrm{Sp} 4$ proteins. Mol Pharmacol 68: 317-329, 2005.

23. Zhou L, Wang DS, Li QJ, Sun W, Zhang Y and Dou KF: The down-regulation of Notch1 inhibits the invasion and migration of hepatocellular carcinoma cells by inactivating the cyclooxygenase-2/Snail/E-cadherin pathway in vitro. Dig Dis Sci 58 : 1016-1025, 2013

24. Que W, Li S and Chen J: NS-398 enhances the efficacy of bortezomib against RPMI8226 human multiple myeloma cells. Mol Med Rep 7: 1641-1645, 2013.

25. Galamb O, Spisák S, Sipos F, et al: Reversal of gene expression changes in the colorectal normal-adenoma pathway by NS398 selective COX2 inhibitor. Br J Cancer 102: 765-773, 2010.

26. Evensen NA, Kuscu C, Nguyen HL, et al: Unraveling the role of KIAA1199, a novel endoplasmic reticulum protein, in cancer cell migration. J Natl Cancer Inst 105: 1402-1416, 2013.

27. Jia S, Ji J and Jiang WG: KIAA1199 knockdown attenuate cell growth of gastric cancer cells and its over-expression is associated with disease progression in patient with gastric cancer. Eur J Cancer 45 (Suppl 2): S575, 2013.
28. Usami S, Wagatsuma M, Fukuoka H, et al: The responsible genes in Japanese deafness patients and clinical application using Invader assay. Acta Otolaryngol 128: 446-454, 2008

29. Dash DP, Silvestri G and Hughes AE: Fine mapping of the keratoconus with cataract locus on chromosome $15 \mathrm{q}$ and candidate gene analysis. Mol Vis 12: 499-505, 2006.

30. Chivu Economescu M, Necula LG, Dragu D, et al: Identification of potential biomarkers for early and advanced gastric adenocarcinoma detection. Hepatogastroenterology 57: 1453-1464, 2010.

31. LaPointe LC, Pedersen SK, Dunne R, et al: Discovery and validation of molecular biomarkers for colorectal adenomas and cancer with application to blood testing. PLoS One 7: e29059, 2012.

32. Paunu N, Lahermo P, Onkamo P, et al: A novel low-penetrance locus for familial glioma at $15 \mathrm{q} 23-\mathrm{q} 26.3$. Cancer Res 62: 3798-3802, 2002.

33. Zweemer RP, Ryan A, Snijders AM, et al: Comparative genomic hybridization of microdissected familial ovarian carcinoma: two deleted regions on chromosome $15 \mathrm{q}$ not previously identified in sporadic ovarian carcinoma. Lab Invest 81: 1363-1370, 2001.

34. Stanton SE, Shin SW, Johnson BE and Meyerson M: Recurrent allelic deletions of chromosome arms $15 \mathrm{q}$ and $16 \mathrm{q}$ in human small cell lung carcinomas. Genes Chromosomes Cancer 27: 323-331, 2000

35. Chanthammachat $\mathrm{P}$, Promwikorn $\mathrm{W}$, Pruegsanusak $\mathrm{K}$, et al: Comparative proteomic analysis of oral squamous cell carcinoma and adjacent non-tumour tissue from Thailand. Arch Oral Biol 58: 1677-1685, 2013.

36. Raish M, Khurshid M, Ansari MA, et al: Analysis of molecular cytogenetic alterations in uterine leiomyosarcoma by arraybased comparative genomic hybridization. J Cancer Res Clin Oncol 138: 1173-1186, 2012.

37. Motaln H, Gruden K, Hren M, et al: Human mesenchymal stem cells exploit the immune response mediating chemokines to impact the phenotype of glioblastoma. Cell Transplant 21: 1529-1545, 2012.

38. Raymond F, Métairon S, Kussmann M, et al: Comparative gene expression profiling between human cultured myotubes and skeletal muscle tissue. BMC Genomics 11: 125, 2010.

39. Harada $\mathrm{H}$ and Takahashi M: CD44-dependent intracellular and extracellular catabolism of hyaluronic acid by hyaluronidase-1 and -2. J Biol Chem 282: 5597-5607, 2007.

40. Csoka AB, Frost GI and Stern R: The six hyaluronidase-like genes in the human and mouse genomes. Matrix Biol 20: 499-508, 2001.

41. Yoshida H, Nagaoka A, Kusaka-Kikushima A, et al: KIAA1199, a deafness gene of unknown function, is a new hyaluronan binding protein involved in hyaluronan depolymerization. Proc Natl Acad Sci USA 110: 5612-5617, 2013.

42. Yoshida H, Nagaoka A, Nakamura S, Sugiyama Y, Okada Y and Inoue S: Murine homologue of the human KIAA1199 is implicated in hyaluronan binding and depolymerization. FEBS Open Bio 3: 352-356, 2013.

43. Yoshida H, Nagaoka A, Nakamura S, Tobiishi M, Sugiyama $Y$ and Inoue S: N-terminal signal sequence is required for cellular trafficking and hyaluronan-depolymerization of KIAA1199. FEBS Lett 588: 111-116, 2013. 